\title{
A Troponina como Marcador de Injúria Celular Miocárdica
}

\author{
Moacir Fernandes de Godoy, Domingo Marcolino Braile, José Purini Neto
}

\author{
São José do Rio Preto, SP
}

\begin{abstract}
A isquemia miocárdica grave, que ocorre nos casos de infarto agudo do miocárdio ou em alguns pacientes com angina instável, resulta em injúria seguida pela liberação de constituintes celulares na corrente sangüínea. Assim sendo, na prática clínica, elevações das taxas da isoforma miocárdica da creatinoquinase (CK-MB) e da desidrogenase láctica (DHL) são interpretadas como marcadores de dano celular miocárdico. A avaliação da atividade dessas enzimas pode ser feita rapidamente e a baixo custo e em situações de rotina são parâmetros satisfatórios para confirmar o diagnóstico, monitorar a evolução e estimar o tamanho do infarto do miocárdio (IM).

Existem limitações uma vez que a especificidade fica comprometida os casos de acometimento muscular esquelético associado e, além disso, a sensibilidade é baixa nas primeiras horas de evolução devido ao aparecimento retardado desses marcadores no sangue. Acrescente-se que a sensibilidade da CK-MB não é suficientemente alta para detectar pequeno dano miocárdio, face à imprecisão analítica das medidas de atividade e à ampla faixa de normalidade ${ }^{1}$.

Isto levou à procura de outros métodos ou novos marcadores diagnósticos de lesão celular miocárdica, tentando superar as limitações.

A mioglobina, uma proteína heme de baixo peso molecular que se encontra anormalmente elevada já nas primeiras $2 \mathrm{~h}$ pós-infarto agudo do miocárdio (IAM) não tem cárdio-especificidade, elevando-se também após lesões musculares esqueléticas e além disso pode causar resultados falso-positivos em portadores de insuficiência renal aguda (eliminação renal).

A proteína ligada a ácidos graxos (H-FABP), que compreende 15 a $30 \%$ de todas as proteínas citoplasmáticas se assemelha à mioglobina com respeito às variações de concentração sérica, tendo especificidade levemente maior. Kleine e $\mathrm{col}^{2}$ compararam os níveis plasmáticos de H-FABP com os de CK-MB e de alfa-hidroxibutirato-desidrogenase (alfa-HBDH), em pacientes com quadro de IAM. Os valores normais máximos de H-FABP $(19 \mu \mathrm{g} / \mathrm{L})$, CK-MB (10U/L) e alfa-HBDH (160U/L) obtidos na dosagem do plasma de 72 doadores de sangue serviram como valor limite para compa-
\end{abstract}

Hospital Português de Beneficência de São José do Rio Preto

Correspondência: Domingo Marcolino Braile - Av. Juscelino Kubitscheck, 3101 - 15091-450 - São José do Rio Preto, SP

Recebido para publicação em 6/2/98

Aceito em 8/7/98 ração. Os níveis de H-FABP foram significativamente maiores que a taxa limite normal com $3 \mathrm{~h}$ de evolução, com picos máximo de H-FABP, CK-MB e alfa-HBDH alcançados com $4,1 \pm 0,9 \mathrm{~h}, 8,4 \pm 1,4 \mathrm{~h}$ e $25,0 \pm 9,5 \mathrm{~h}$ após o IAM, respectivamente, indicando que a H-FABPé mais adequada que a CK-MB e a alfa-HBDH para o diagnóstico precoce daquela afecção.

A glicogeno-fosforilase também é uma proteína cistosólica e com grande cárdio-especificidade com cinética de sua isoforma BB similar à da mioglobina. A experiência clínica com a utilização desse marcador ainda é limitada, precisando ser confirmada em séries maiores. Mair e $\mathrm{col}^{3}$ procuraram determinar se as alterações transitórias de ST-T em pacientes com angina instável (sem IM pelos critérios padrão), estavam ou não associadas com elevação da glicogeno-fosforilase BB, por ocasião da admissão hospitalar, chegando à conclusão de que aquela enzima foi o único marcador que esteve significativamente acima de seu valor discriminatório na maioria dos pacientes $(\mathrm{p}=0,0001)$. Concluíram que a liberação precoce de glicogeno-fosforilase $\mathrm{BB}$ pode ajudar a identificar pacientes de alto risco com angina instável, mesmo no atendimento emergencial.

Recentemente, as troponinas têm recebido crescente atenção como marcadores altamente específicos de injúria celular. As troponinas formam um complexo que regula a interação cálcio-dependente da miosina com a actina. São constituídas de três diferentes proteínas (troponina I, Ce T) existentes tanto no músculo esquelético quanto cardíaco e codificadas por diferentes genes. A troponina Cé idêntica tanto no músculo esquelético como cardíaco mas os genes codificadores das troponinas I e T, cardíaca e esquelética, são diferentes, o que permitiu que anticorpos monoclonais de reatividade cruzada extremamente baixa pudessem ser desenvolvidos facilitando o diagnóstico do IAM. Em pacientes com IAM, a elevação da atividade da creatinofosfoquinase acima dos valores normais é raramente encontrada de 4 a 6 h após o início da dor, fazendo com que o diagnóstico precoce tenha que depender fortemente de alterações eletrocardiográficas típicas. Isto torna-se um problema pelo fato de que o eletrocardiograma (ECG) é inconclusivo em até $40 \%$ dos pacientes.

A troponina I cardíaca não se expressa no músculo esquelético humano durante o desenvolvimento fetal, após trauma do músculo esquelético ou durante a regeneração desse tipo de músculo. Ao contrário da CK-MB, a troponina I cardíaca é altamente específica para o tecido miocárdico, nãoé detectável no sangue de pessoas sadias, mostra um aumento proporcionalmente bem maior acima dos valores limite, 
nos casos de IM e pode permanecer elevada por sete a 10 dias após o episódio agudo ${ }^{4,5}$. Esse fato foi valorizado por Fonarow ${ }^{5}$ ao implementar um ensaio com a troponina I na avaliação de dor precordial em pacientes que procuravam o serviço de urgência do UCLA Medical Center. Com auxílio desse teste foi possível excluir, com grande segurança, a existência de IM e em menos da metade do tempo que com a abordagem tradicional utilizando a CK-MB.

A troponina I e a troponina T tornam-se mensuráveis 3 a 4h após o início do IM. Estudos dos níveis pico de troponina $\mathrm{T}$ medidos dentro das primeiras $24 \mathrm{~h}$ após a admissão em pequenos grupos selecionados de pacientes com dor precordial têm demonstrado um excesso de eventos cardíacos nos pacientes com elevação de troponina $\mathrm{T}$, mesmo naqueles sem elevação da CK-MB ${ }^{6}$. Hamm e col $^{7}$ investigaram de forma prospectiva a utilidade da dosagem das troponinas T e I na avaliação de pacientes com dor precordial aguda. Para tanto acompanharam 773 pacientes que haviam apresentado dor precordial com menos de $12 \mathrm{~h}$ mas sem elevação do segmento ST ao ECG. Verificaram que a troponina T foi positiva em $123(16 \%)$ pacientes e a troponina I em 171 (22\%). Entre os 47 pacientes que evoluíram para IM, a troponina T foi positiva em 44 (94\%) e a troponina I foi positiva em todos os 47 . Entre os 315 pacientes com angina instável, a positividade das troponinas $\mathrm{T}$ e I foi respectivamente de $22 \%$ e $36 \%$. Ambas as troponinas provaram ser, de forma independente, fortes preditores de eventos cardíacos. A taxa de eventos, morte ou infarto não fatal, nos pacientes com testes negativos foi extremamente baixa (1,1 e 0,3 para troponinas T e I, respectivamente). Lüscher e $\mathrm{col}^{8}{ }^{8}$ também procuraram determinar a aplicabilidade das troponinas T e I na estratificação de risco de pacientes com doença coronária instável, concluindo que ambas propiciam informação prognóstica independente em relação à morte e IM. A capacidade preditiva dos marcadores variou de acordo com o nível de corte, mas já foi significante com valores de $0,05 \mu \mathrm{g} / \mathrm{L}$ para a troponina $\mathrm{Te}$ 1,5 $\mu \mathrm{g} / \mathrm{L}$ para a troponina I. Afirmam que estudos prospectivos poderão indicar se a troponina $\mathrm{T}$ e a troponina I serão capazes de identificar pacientes que se beneficiarão de tratamento antitrombótico e/ou procedimentos invasivos.

Mair e $\operatorname{col}^{9}$ demonstraram que o primeiro sinal de elevação das concentrações de troponina em pacientes com IM ocorria já com 3,5h de evolução, em 50\% dos casos, sendo necessárias 4,75h, em média, para se obter a mesma taxa de comprometimento com a CK-MB. Com 7h de evolução, 95\% dos pacientes apresentavam alteração da troponina, fato só igualado com a CK-MB após $12 \mathrm{~h}$ de início dos sintomas. Uma das grandes vantagens da dosagem de troponina ao invés de $\mathrm{CK}-\mathrm{MB}$ é que aquela atinge valores pico de até mais de 40 vezes o limite de detecção, enquanto esta se restringe a seis a nove vezes. Sabe-se também que tanto a troponina I quanto a troponina $\mathrm{T}$ apresentam sensibilidade equivalente para o diagnóstico de injúria celular miocárdica ${ }^{10}$. Wu e col ${ }^{11}$ mediram de forma seriada as concentrações plasmáticas de mioglobina, CK-MB e troponina I em 25 pacientes com diagnóstico confirmado de IAM e em 74 pa- cientes com suspeita de infarto mas nos quais esse diagnóstico foi posteriormente descartado. $\mathrm{O}$ valor de corte na concentração de troponina I foi determinado como sendo 2,5ng/mL. Dos três marcadores, a mioglobina foi a que apresentou maior sensibilidade (50\%) quando o sangue foi coletado nas primeiras $6 \mathrm{~h}$ após o início da dor. Todos os marcadores usados mostraram alta sensibilidade (>93\%), 6 a $24 \mathrm{~h}$ após o início dos sintomas. A CK-MB permaneceu mostrando alta sensibilidade por 48h, enquanto que a troponina I foi bastante sensível até $72 \mathrm{~h}$. Entre 72 e $150 \mathrm{~h}$ a troponina I ainda mostrava sensibilidade de $70 \%$ enquanto que com a mioglobina e a CK-MB a sensibilidade foi de $21 \%$ e $18 \%$, respectivamente. A especificidade da troponina I para pacientes sem confirmação de IM foi equivalente à da CK-MB e significativamente maior que para a mioglobina. A especificidade da troponina I em 59 pacientes com insuficiência renal crônica, doenças musculares esqueléticas ou trauma muscular foi maior que a de todos os outros marcadores incluindo a troponina T. Jaffe e $\mathrm{col}^{12}$ procuraram as sensibilidades relativas da troponina I e das isoenzimas da desidrogenase láctica ao longo do tempo no diagnóstico de IM. Comprovaram que a troponina I era no mínimo tão sensível quanto as isoenzimas da desidrogenase láctica, uma vez que $90 \%$ dos pacientes com IAM ainda mantinham concentrações de troponina I acima do normal mesmo no $4^{\circ}$ dia pós internação na Unidade Coronária. Bertinchant e col ${ }^{13}$ verificaram que em pacientes admitidos na Unidade de Terapia Intensiva com quadro de IAM, a troponina I esteve elevada em todos os casos e a detecção foi mais precoce após o início da dor $(4,5 \pm 2,3 \mathrm{~h})$ que para a CK-MB $(6,3 \pm 3,6 \mathrm{~h}$; $\mathrm{p}=0,003)$. Os valores pico para troponina I e CK-MB ocorreram $12,2 \pm 4,6 \mathrm{~h}$ e $15,8 \pm 9,0 \mathrm{~h}$, respectivamente, nos pacientes tratados com trombólise e o desaparecimento plasmático da troponina I aconteceu entre 5 e 9 dias após o início da dor, bem mais tarde que para a CK-MB $(\mathrm{p}=0,0001)$. Puderam ainda documentar em 49 pacientes submetidos à trombólise, que a sensibilidade comparativa dos dois testes, admitindose valores normais limite de $0,1 \mathrm{ng} / \mathrm{mL}$ para a troponina e 15 IU/L para CK-MB, com base na $1^{\mathrm{a}}$ amostra colhida (em média $3,4 \pm 1,3 \mathrm{~h}$ após o início da dor), foi de $61 \%$ e $22 \%$, respectivamente ( $\mathrm{p}=0,0002)$. A troponina I não foi detectada no plasma de 145 indivíduos normais nem em qualquer um dos seis pacientes com trauma muscular grave ou rabdomiólise, resultando portanto em uma especificidade de $100 \%$. Mair e $\mathrm{col}^{14}$ demonstraram que a liberação de troponina I em pacientes com IAM correlaciona-se com o tamanho do infarto. Em um grande grupo de pacientes admitidos para avaliação de suspeita de IAM a sensibilidade da troponina T (64\%) foi maior que a de outros marcadores, como a atividade de $\mathrm{CK}$ MB e o nível de mioglobina. A especificidade porém, foi mais baixa que a da CK-MB e da mioglobina devido aos $37 \%$ de resultados falso-positivos em pacientes com angina instável. Em pacientes com injúria muscular esquelética as troponinas são superiores ao CK na indicação de envolvimento cardíaco, já que são específicas para comprometimento miocárdico. Em adição, devido ao seu tempo prolongado de liberação, as troponinas I e T permitem confirmação 
do diagnóstico de infarto até três semanas após o início dos sintomas ${ }^{6}$.

Também já foi demonstrado o valor prognóstico da troponina em pacientes com angina instável. Wu e col ${ }^{15}$ procuraram demonstrar o valor prognóstico a curto prazo (três semanas), da dosagem da troponina-T e da CK-MB logo na admissão de pacientes com angina instável em um serviço de emergência. De um grupo de 486 pacientes com dor precordial, selecionaram 131 com quadro clínico de angina instável, nos quais fizeram dosagens de CK-MB e troponina-T com valores de corte de 5 a $10 \mathrm{ng} / \mathrm{mL}$ para o caso da CK-MB e de 0,1 e 0,2ng/mL para a troponina-T. Considerando o valor de corte em $0,1 \mathrm{ng} / \mathrm{mL}$, a troponina-T estava elevada em 27 dos 131 pacientes com angina instável enquanto que a $\mathrm{CK}-\mathrm{MB}$ estava anormal em apenas oito (6\%) casos, seja com valor de corte de $10 \mathrm{ng} / \mathrm{mL}$ seja com valor de corte de $5 \mathrm{ng} / \mathrm{mL}$ e todos esses oito estavam com a troponina-T elevada. O risco relativo da elevação de troponina-T foi de 14 (IC95\% 3,4-58) para a ocorrência de IM e de 33 (IC95\% 4,3-251) para a ocorrência de qualquer evento maior (infarto, revascularização ou angina refratária). O valor de corte de $0,2 \mathrm{ng} / \mathrm{mL}$ para troponina- $\mathrm{T}$ não alterou significativamente os resultados. É importante ressaltar que características clínico-epidemiológicas não se correlacionaram com a elevação da troponina- $T$, sugerindo que o teste provê informação adicional, podendo indicar tratamento mais agressivo ou invasivo nos portadores de angina instável com troponina-T elevada à admissão.

Em um estudo de 84 pessoas com angina de repouso e sem elevação da CK-MB, valores normais de troponina se correlacionaram com baixa probabilidade de má evolução $(1 / 51=1,9 \%)$ enquanto que os valores alterados prognosticaram eventos adversos $(10 / 33=30,3 \%)$. Deste modo, valores alterados de troponina são capazes de denunciar a presença de micro-infartos não diagnosticados pela CK$\mathrm{MB}$, permitindo aos médicos identificar quais pacientes podem se beneficiar de uma terapêutica mais intensiva e eventualmente de um procedimento invasivo ${ }^{5}$.

Antman e col ${ }^{16}$ estudaram o valor prognóstico dos níveis de troponina I em 1404 pacientes com angina instável ou infarto não-Q. A mortalidade aos 42 dias foi significativamente maior nos 573 pacientes com níveis de troponina I de pelo menos $0,4 \mathrm{ng} / \mathrm{mL}$ ( 21 mortes ou $3,7 \%$ ) que nos 831 pacientes com troponina I abaixo de $0,4 \mathrm{ng} / \mathrm{mL}$ (oito mortes ou $1,0 \% ; \mathrm{p}<0,001)$.

Tem sido relatado que a cardioversão elétrica pode se seguir de alterações enzimáticas. Para estudar o assunto, Bonnefoy e $\mathrm{col}{ }^{17}$ mediram a troponina I sérica, mioglobina, CK total e CK-MB 1, 2, 3, 4, 8, 12 e 24h após cardioversão eletiva de taquicardia supraventricular em 28 pacientes (oito mulheres e 20 homens; idade média de $64 \pm 10$ anos). A energia cumulativa foi abaixo de $370 \mathrm{~J} \mathrm{em} 17$ pacientes, entre 370 e 900 em oito e $1020 \mathrm{~J}$ em três. O limite de detecção do teste utilizado era de $0,35 \mu \mathrm{g} / \mathrm{L}$ com faixa de normalidade de 0,35 a $1,3 \mu \mathrm{g} / \mathrm{L}$. A troponina I se manteve dentro da normalidade em todos os casos e não foi notada correlação entre os níveis de troponina I e o número ou energia da cardioversão empregada. A mioglobina e a CK aumentaram até concentrações anormais em 11 pacientes (mioglobina $630 \pm 190 \mu \mathrm{g} / \mathrm{L}$ e CK total de 2584 $\pm 780 \mathrm{U} / \mathrm{L}$ ), alcançando níveis diagnósticos de IM em cinco pacientes. Houve forte correlação entre a energia total da cardioversão e os níveis de mioglobina e CK total, sugerindo que as alterações verificadas se devam exclusivamente ao comprometimento muscular esquelético e não cardíaco, já que não houve alteração da troponina I. Georges e col ${ }^{18}$ também já haviam documentado que apenas a troponina I não se elevou em 25 pacientes submetidos a cardioversão elétrica.

Portadores de miocardite freqüentemente necessitam de biópsia miocárdica na tentativa de adequada definição diagnóstica. A necessidade desse procedimento invasivo e de certa morbidade limitam a realização em larga escala. Como o diagnóstico histológico de miocardite requer a presença de lesão do miócito, Smith e col ${ }^{19}$ procuraram verificar se a dosagem de troponina I cardíaca poderia ajudar no diagnóstico. Primeiramente, para validação do método, dosaram a troponina em 26 ratos com miocardite auto-imune. Em 24 deles a troponina esteve elevada, permanecendo normal em todos os animais do grupo controle $(\mathrm{p}<0,001)$. Em seguida, a troponina I foi medida no soro de 88 pacientes envolvidos no Myocarditis Treatment Trial, comparando-se com as dosagens de CK-MB. A troponina I esteve elevada em 18 (34\%) de 53 pacientes com miocardite e em somente quatro $(11 \%)$ dos 35 pacientes sem miocardite $(\mathrm{p}=0,01)$. Em contraste, os valores de CK-MB estiveram elevados em apenas três $(5,7 \%)$ dos 53 pacientes com miocardite e em nenhum dos pacientes sem miocardite $(\mathrm{p}=0,27)$. Deste modo, as elevações da troponina I ocorreram mais freqüentemente que as alterações da CK-MB em pacientes com miocardite comprovada $(\mathrm{p}=0,001)$ e além disso os níveis de troponina I correlacionaram-se com a duração dos sintomas de insuficiência cardíaca, alertando para o fato de que a maior parte da necrose dos miócitos ocorre na fase inicial do processo inflamatório e que, portanto, o diagnóstico e o tratamento precisam ser realizados o mais breve possível.

A cirurgia cardíaca exige na grande maioria das vezes que o coração permaneça estático e exangue enquanto se realiza o procedimento. Para tanto são utilizados os circuitos de circulação extracorpórea que dão o suporte cardíaco e pulmonar durante o tempo operatório. Já está bem documentado que durante esse tempo de parada, o coração sofre um processo de isquemia controlada que culmina em maior ou menor quantidade de morte tecidual (infarto), sendo este o motivo da utilização das soluções cardioprotetoras durante a cardioplegia tentando minimizar as conseqüências sobre a função cardíaca. O diagnóstico de infarto peri-operatório muitas vezes é difícil tanto pela sedação do paciente e intubação, impedindo a comunicação verbal de sintomas, como pela baixa sensibilidade e especificidade fornecida pelos monitores eletrocardiográficos comuns restritos, na maioria das vezes, a apenas uma ou no máximo duas derivações. Maie e $\mathrm{col}^{20}$ utilizaram a troponina I para avaliar a ocorrência de IM peri-operatório em pacientes submetidos à revascularização miocárdica com pontes de safena. Estuda- 
ram 28 pacientes ( 26 eletivos e 2 de emergência), antes e após a revascularização. A troponina I elevou-se em 22 dos 26 pacientes eletivos, os quais não mostravam evidência clínica ou laboratorial de IM refletindo com isso o inevitável dano miocárdico causado pela parada cardioplégica. $\mathrm{O}$ valor pico nesses casos foi de 1,7 $71,0 \mu \mathrm{g} / \mathrm{L}$. Dois dos $22 \mathrm{sem}$ infarto peri-operatório mostraram níveis de troponina I acimade 3,0 $\mu \mathrm{g} / \mathrm{L}(3,9$ e $3,4 \mu \mathrm{g} / \mathrm{L})$ indicando maior comprometimento miocárdico que nos outros 20 , embora sem elevação significante da CK-MB. Quatro dos 26 pacientes eletivos mostraram quadro eletrocardiográfico, ecocardiográfico e enzimático (CK-MB) de infarto peri-operatório. Um deles, com infarto $\mathrm{Q}$, apresentou pico de troponina I de aproximadamente $30 \mu \mathrm{g} / \mathrm{L}$ e três com infarto não-Q mostraram níveis em torno de 5,0 $\mathrm{g} / \mathrm{L}$. Os autores sugerem que, após operações eletivas de revascularização miocárdica, picos de troponina I acima de $3,7 \mu \mathrm{g} / \mathrm{L}$, concentrações acima de $3,1 \mu \mathrm{g} / \mathrm{L}$ após $12 \mathrm{~h}$ ou concentrações em torno de $2,5 \mu \mathrm{g} / \mathrm{L}$ após $24 \mathrm{~h}$ indicam IM peri-operatório com alta probabilidade. Etievent e $\operatorname{col}^{21}$ mediram a liberação de troponina I em dois grupos de pacientes após cirurgia cardíaca (troca de valva aórtica versus revascularização miocárdica). Os valores médios da troponina I foram mais elevados no grupo de pacientes submetidos à revascularização miocárdica. No grupo de pacientes submetidos à troca valvar aórtica foi encontrada correlação positiva entre o tempo de clampeamento aórtico e os níveis de troponina I. Concluem que a troponina I é um marcador adequado da isquemia miocárdica durante operações cardíacas e pode ser usado para avaliar procedimentos de cardioproteção. Brackenbury e $\mathrm{col}^{22}$ demonstraram que os níveis de troponina foram menores em pacientes submetidos à cirurgia cardíaca com soluções cardioplégicas aquecidas em detrimento das soluções cardioplégicas geladas, concluindo que as soluções aquecidas devem proporcionar melhor proteção miocárdica durante $\mathrm{o}$ ato cirúrgico. Chocron e $\mathrm{col}^{23}$ estudaram 60 pacientes divididos aleatoriamente em dois grupos, um recebendo solução cardioplégica apenas por via anterógrada e o outro por via anterógrada seguida de via retrógrada. Verificaram que a utilização da solução cardioplégica cristalóide por via anterógrada provocou melhor proteção em termos de menor liberação de troponina I, que somente por via anterógrada, nos pacientes com estenose do tronco coronário esquerdo. Taggarte col analisaram a vulnerabilidade miocárdica em crianças submetidas a cirurgia cardíaca no sentido de confirmar as informações de que em animais jovens o miocárdio é mais resistente à isquemia e à reperfusão do que o miocárdio adulto. Para tanto analisaram, prospectivamente, os marcadores de injúria miocárdica (mioglobina, CK-MB, troponina T e troponina I), em uma população pediátrica de 40 pacientes submetida a operações intracardíacas ou não. As dosagens foram realizadas com 1, 6, 24 e 48 a 72h após a operação. Encontraram elevações significantes da mioglobina e da CK-MB, mas não das troponinas T e I no grupo controle (operações extracardíacas). Houve elevação significante dos marcadores em todos os pacientes submetidos a opera- ções intracardíacas, especialmente nos portadores de comunicação interventricular e transposição dos vasos da base. Os aumentos na CK-MB e na troponina $\mathrm{T}$ foram cerca de cinco vezes maiores que os encontrados em pacientes adultos, sugerindo que, na verdade, o miocárdio de criança possa ser mais vulnerável à injúria durante cirurgia cardíaca, que o miocárdio de adultos.

Em pacientes criticamente doentes pode haver comprometimento cardíaco não reconhecido. Guest e $\mathrm{col}^{25}$ procuraram determinar a importância dessa ocorrência e para tanto desenvolveram um estudo cego, prospectivo nas unidades de terapia intensiva de um centro médico acadêmico. Foram avaliados 209 pacientes com dosagem diária de troponina I. Desse grupo, $32(15,0 \%)$ apresentaram evidências de dano miocárdio com base nos níveis elevados de troponina I. Somente em 12 (37.0\%) desses 32 pacientes havia sido feito o diagnóstico de IAM, sendo que deixou de ser reconhecido clinicamente nos outros 20 . O comprometimento não reconhecido foi mais comum nos jovens e nos negros. A mortalidade nos pacientes com comprometimento miocárdico reconhecido foi de $42,0 \%$ no não reconhecidos e maior do que naqueles sem comprometimento $(15,0 \%$; $\mathrm{p}<0,001)$. Os pacientes com alteração cardíaca encontravam-se freqüentemente hipotensos ( $75,0 \%$ versus $50,0 \%$; $\mathrm{p}=0,007)$, mais necessitados de ventilação mecânica $(66,0 \%$ versus $27,0 \%$; $\mathrm{p}<0,001)$ e tiveram maior tempo de permanência na UTI $(5,3$ versus 3,1 dias; $p<0,007)$ que os sem comprometimento cardíaco, constatando-se dessa forma que a incidência de lesão miocárdica, em pacientes gravemente enfermos em UTI e definida por níveis elevados de troponina I, é inesperadamente alta e associada a um aumento de morbidade e mortalidade. Ressalta o fato de não haver reconhecimento clínico na maioria dos casos. Hirsch e $\mathrm{col}^{26} \mathrm{demonstraram} \mathrm{em} \mathrm{crian-}$ ças que a dosagem de troponina Ié o que mais se aproxima, na atualidade, do que poder-se-ia chamar de "padrão ouro" para detecção de lesão miocárdica. Towbin ${ }^{27}$, em editorial sobre o assunto, considerou o trabalho de Hirsch e col como um importante avanço, provendo a comunidade médica pediátrica com um melhor método de avaliação de lesões cardíacas específicas em crianças. Lipshultz e $\mathrm{col}^{28}$ investigaram a utilidade da dosagem de troponina $\mathrm{T}$ em 51 pacientes com idades variando de um dia a 34 anos (média 5,7 anos) submetidos a cirurgia cardiovascular (19 casos), outras cirurgias que não a cardiovascular (17 casos) ou que haviam recebido doxorubicina em decorrência de leucemia linfoblástica aguda ( 15 casos). Admitindo como valor mínimo detectável $0,03 \mathrm{ng} / \mathrm{mL}$, verificaram que a troponina T estava elevada em crianças, de todas as idades, com dano miocárdico. Notaram correlação entre um escore de gravidade cirúrgica e os níveis pós-operatórios de troponina $\mathrm{T}(\mathrm{p}<0,0001)$. Também verificaram que a magnitude da elevação da troponina $T$ em pacientes que receberam doxorubicina foi capaz de predizer a ocorrência de dilatação ventricular esquerda e adelgaçamento da parede ventricular, nove meses mais tarde. Kaplan ${ }^{29}$,em editorial sobre esse trabalho, ratifica a importância da avaliação dos níveis séricos de troponina em crianças com dano miocárdico declarado ou potencial. 
Existe, finalmente, aplicação para dosagem seriada de troponina em pacientes com trauma e suspeita de contusão cardíaca. Adams e $\mathrm{col}^{30}$, procurando determinar se a dosagem de troponina I poderia melhorar a capacidade de detecção de lesão cardíaca em pacientes com trauma torácico, estudaram 44 pacientes com ecocardiograma seriado e dosagens laboratoriais. Seis pacientes tinham evidência de lesão cardíaca pelo ecocardiograma, sendo que todos mostraram elevação da CK-MB e da troponina I. Dos 37 pacientes sem evidências ecocardiográficas de contusão cardíaca, 26 ti- nham elevação da CK-MB mas nenhum mostrou elevação da troponina I. Concluem que a dosagem da troponina I detecta acuradamente a lesão cardíaca em pacientes com trauma torácico, facilitando com isso o diagnóstico e o tratamento desses pacientes.

Com base no relatado na literatura, a introdução da dosagem de troponinas na prática clínica diária dos vários centros cardiológicos deverá facilitar enormemente o diagnóstico e a condução dos casos duvidosos e a melhor avaliação do grau de sofrimento miocárdico após eventos clínicos ou procedimentos cirúrgicos.

\section{Referências}

1. Hamm CW, Katus HA-New biochemical markers for myocardial cell injury. Curr Opin Cardiol 1995; 10: 355-60.

2. Kleine AH, Glatz JFCF, Van Nieuwenhoven FA, Van der Vusse GJ - Release of heart fatty acid-binding protein into plasma after acute myocardial infarction in man. Mol Cell Biochem 1992; 116: 155-62.

3. Mair J, Puschendorf B, Smidt J et al - Early release of glycogen phosphorylase in patients with unstable angina and transient ST-T alterations. Br Heart J 1994; 72: 125-7.

4. Antman EM, Tanajisevic MJ, Thompson B et al-Cardiac specific troponin I levels to predict the risk of mortality in patients with acute coronary syndromes. N Engl J Med 1996; 335: 1342-9.

5. Fonarow GC, UCLA Clinical Practice Guideline - Cardiac Troponin I Assay Diagnostic Module. July, 1996; 2.

6. Ohman EM, Armstrong PW, Christenson RH et al - Cardiac troponin T levels for risk stratification in acute myocardial ischemia. N Engl J Med 1996; 335: 1333-41.

7. Hamm CW, Goldmann BU, Heeschen C, Kreymann G, Berger J, Meinertz T Emergency room triage of patients with acute chest pain by means of rapid testing for cardiac troponin T or troponin I. N Engl J Med 1997; 337: 1648-53.

8. Lüsher MS, Thygesen K, Ravkilde J, Heickendorff J - Applicability of cardiac troponin $\mathrm{T}$ and $\mathrm{I}$ for early risk stratification in unstable coronary artery disease. Circulation 1997; 96: 2578-85.

9. Mair J, Wagner I, Jakob G et al - Different time courses of cardiac contractile proteins after acute myocardial infarction. Clin Chim Acta 1994; 231: 47-60.

10. Mair J, Morandell D, Genser N, Lechleitner P, Dienstl F, Puschendorf B Equivalent early sensitivities of myoglobin, creatine kinase MB mass, creatine kinase isoform ratios, and cardiac troponins I and $\mathrm{T}$ for acute mtocardial infarction. Clin Chem 1995a; 41: 1266-72.

11. Wu AH, Feng YJ, Contois JH, Pervaiz S - Comparison of myoglobin, creatine kinase-MB, and cardiac troponin I for diagnosis of acute myocardial infarction. Ann Clin Lab Sci 1996; 26: 291-300.

12. Jaffe AS, Landt Y, Parvin CA, Abendschein DR, Geltman EM, Landenson JH Comparative sensitivity of cardiac troponin I and lactate dehydrogenase isoenzimes for diagnosing acute myocardial infarction. Clin Chem 1996; 42: 1770-6.

13. Bertinchant JP, Larue C, Pernel I et al - Interet du dosage de la troponine humaine dans le diagnostic de l'infarction aigu du myocarde. Arch Mal Couer Vaiss 1996; 89: 63-8.

14. Mair J, Wagner I, Morass B et al - Cardiac troponin I release correlates with myocardial infarction size. Eur J Clin Chem Biochem 1995b; 33: 869-72.
15. Wu AHB, Abbas AS, Green S et al-Prognostic value of cardiac troponin $\mathrm{T}$ in unstable angina pectoris. Am J Cardiol 1995; 76: 970-2.

16. Antman EM, Tanasijevic MJ, Thompson B et al-Cardiac specific troponin I levels to predict the risk of mortality in patients with acute coronary syndromes. N Engl J Med 1996; 335: 1342-9.

17. Bonnefoy E, Chevalier P, Kirkorian G, Guidolet J, Marchand A, Toubvoul P Cardiac troponin I does not increase after cardioversion. Chest 1997; 111: 15-8.

18. Georges JL, Spentchian M, Caubel C et al - Time course of troponin I, myoclobulin, and cardiac enzyme release after electrical cardioversion. Am J Cardiol 1996; 78: 825-6

19. Smith SC, Ladenson JH, Mason JW, Jaffe AS - Elevations of cardiac troponin I associated with myocarditis. Experimental and clinical correlates. Circulation 1997; 95: 163-8.

20. Mair J, Larue C, Mair P, Balogh D, Calzolari C, Puschendorf B - Use of cardiac troponin I to diagnose perioperative myocardial infarction in coronary artery bypass grafting. Clin Chem 1994c; 40(II Pt I); 2066-70.

21. Etievent JP, Chocron S, Toubin G et al - Use of cardiac troponin I as a marker of perioperative myocardial ischemia. Ann Thorac Surg 1995; 59: 1192-4.

22. Brackenbury ET, Sherwood R, Meehan N et al - Troponin T release with warm and cold cardioplegia. Perfusion 1996; 11: 377-82.

23. Chocron S, Alwan K, Toubin G et al - Crystalloid cardioplegia route of delivery and cardiac tropnin I release. Ann Thorac Surg 1996; 62: 481-5.

24. Taggart DP, Hadjinikolas L, Wong K et al - Vulnerability of paediatric myocardium to cardiac surgery. Heart 1996; 76: 214-7.

25. Guest TM, Ramanathan AV, Tuteur PG, Schechtman KB, Ladenson JH, Jaffe AS Myocardial injury in critically ill patients. A frequently unrecognized complication. JAMA 1995; 273: 1945-9.

26. Hirsch R, Landt $Y$, Porter $S$ et al - Cardiac troponin I in pediatrics: normal values and potential use in the assessment of cardiac injury. J Pediatr 1997; 130: 872-7.

27. Towbin JA - Cardiac troponin IL A new diagnostic gold standard of cardiac injury in children? J Pediatr 1997; 130: 853-5.

28. Lipshultz SE, Rifai N, Sallan SE et al - Predictive value of cardiac troponin T in pediatric patients at risk for myocardial injury. Circulation 1997; 96: 2641-8.

29. Kaplan S - Biochemical markers of myocardial injury in children. Circulation 1997;96: 2496-7.

30. Adams JE $3^{\text {rd }}$, Davila-Roman VG, Bessey PQ, Blake DP, Ladenson JH, Jaffe AS Improved detection of cardiac contusion with cardiac troponin I. Am Heart J 1996; 131: 308-12 